\title{
Imaging of carotid artery vessel wall edema using T2-weighted cardiovascular magnetic resonance
}

\author{
Lars Ølgaard Bloch ${ }^{1,2^{*}}$, Anne Yoon Krogh Grøndal Hansen ${ }^{2,3}$, Steen Fjord Pedersen ${ }^{2,3}$, Jesper Langhoff Honge ${ }^{3}$,
} Won Yong Kim ${ }^{1,2}$ and Esben Søvsø Szocska Hansen²

\begin{abstract}
Background: Atherothrombosis remains a major health problem in the western world, and carotid atherosclerosis is an important contributor to embolic ischemic strokes. It remains a clinical challenge to identify rupture-prone atherosclerotic plaques before clinical events occur. Inflammation, endothelial injury and angiogenesis are features of vulnerable plaques and may all be associated with plaque edema. Therefore, vessel wall edema, which can be detected by 2D T2-weighted cardiovascular magnetic resonance (CMR), may be used as a dynamic marker of disease activity in the atherosclerotic plaque. However, 2D imaging is limited by low spatial resolution in the slice-select direction compared to 3D imaging techniques. We sought to investigate the ability of novel 3D techniques to detect edema induced in porcine carotid arteries by acute balloon injury compared to conventional 2D T2-weighted black-blood CMR.
\end{abstract}

Methods: Edema was induced unilaterally by balloon overstretch injury in the carotid artery of nine pigs. Between one to seven hours (average four hours) post injury, CMR was performed using 2D T2-weighted short-tau inversion recovery (T2-STIR), 3D volumetric isotropic turbo spin echo acquisition (VISTA) and 3D T2 prepared gradient-echo (T2prep-GE). The CMR images were compared in terms of signal-to-noise ratio (SNR) and contrast-to-noise (CNR) ratio. Furthermore, the presence of vessel wall injury was validated macroscopically by means of Evans Blue dye that only enters the injured vessel wall.

Results: All three imaging sequences classified the carotid arteries correctly compared to Evans Blue and all sequences demonstrated a significant increase in SNR of the injured compared to the non-injured carotid vessel wall (T2-STIR, $p=0.002$; VISTA, $p=0.004$; and T2prep-GE, $p=0.003$ ). There was no significant difference between sequences regarding SNR and CNR.

Conclusion: The novel 3D imaging sequences VISTA and T2prep-GE perform comparably to conventional 2D T2-STIR in terms of detecting vessel wall edema. The improved spatial coverage of these 3D sequences may facilitate visualization of vessel wall edema to enable detection and monitoring of vulnerable carotid atherosclerotic plaques.

Keywords: Cardiovascular magnetic resonance, Atherosclerosis, Inflammation, Carotid artery

\section{Background}

Stroke is a leading cause of disability and the fourth leading cause of death in the US [1]. About $87 \%$ of all strokes are ischemic [1], of which carotid atherosclerotic lesions are considered to account for about 25\% [2]. Inflammation plays a key role in all stages of atherosclerosis

\footnotetext{
* Correspondence: LARS.OLGAARD.BLOCH@STUDMED.AU.DK

'Department of Cardiology, Aarhus University Hospital Skejby,

Brendstrupgaardsvej 100, DK-8200 Aarhus N, Denmark

${ }^{2}$ MR Research Centre, Aarhus University Hospital Skejby, Brendstrupgaardsvej

100, DK-8200 Aarhus N, Denmark

Full list of author information is available at the end of the article
}

and subsequently may lead to atherothrombosis [3,4]. Accordingly, on-going research is aimed to find imaging methods that can identify the inflammatory status of the plaque. Several imaging modalities have been used in this respect. Positron emission tomography with detection of arterial fluorodeoxyglucose uptake has been shown to correlate with the amount of macrophages [5-7] in the atherosclerotic plaque providing a measure of the inflammatory burden. Also, carotid inflammation can be detected by contrast-enhanced cardiovascular magnetic resonance using targeted gadolinium agents $[8,9]$ 
or ultra-small particles of iron oxide [10,11]. Tearney et al. showed that the optical coherence tomography signal correlates with the amount of macrophages in the plaque [12]. Other invasive methods that may be able to identify inflammation in atherosclerotic lesions include near-infrared spectroscopy [13] and thermography $[14,15]$. However, it would be preferable to have a non-contrast dependent non-invasive imaging method that may identify the inflammatory status of the plaque to monitor plaque development and response to therapy.

Some of the characteristic features of vulnerable plaques include inflammation, endothelial injury and neoangiogenesis $[3,4,16,17]$ which may all be associated with vessel wall edema. Previous studies have demonstrated that vessel wall edema in the carotid and coronary arteries can be detected by 2D T2-weighted (T2W) short-tau inversion recovery (T2-STIR) $[18,19]$. Furthermore, evidence now exists that vessel wall edema is present and can be detected by 2D T2-STIR within the culprit lesion of the coronary arteries in patients with acute coronary syndrome $[20,21]$. Since $2 D$ sequences are limited by poor spatial resolution and reduced coverage, we sought to determine the ability of 3D T2W black-blood MRI sequences to detect edema induced in porcine carotid arteries by acute balloon injury [22] compared with conventional 2D T2-STIR. Zhang et al. demonstrated in human femoral arteries that 3D turbo spin echo (TSE) with variable flip angles enabled superior vessel wall visualization, superior blood signal suppression, and comparable vessel wall morphological measurements compared to 2D TSE T2W imaging [23]. Thus, we included a Volumetric ISotropic Turbo spin echo Acquisition (VISTA) in the imaging protocol of our study. We also included a 3D T2 prepared gradient-echo (T2prep-GE) sequence with motion sensitized gradients to ensure black-blood properties.

\section{Methods}

Animal model

Nine female Danish Land Race pigs weighing about $40 \mathrm{~kg}$ were used for the experiments. The pigs were treated in accordance with the Danish Law on Animal Experiments. The pigs were pre-sedated with an intramuscular injection of azaperone, $(4 \mathrm{mg} / \mathrm{kg})$ and midazolam $(0,5 \mathrm{mg} / \mathrm{kg})$ which allowed intravenous access via an ear vein. Midazolam $(0,5 \mathrm{mg} / \mathrm{kg})$ and ketamine $(5 \mathrm{mg} / \mathrm{kg})$ were administered intravenously before endotracheal intubation and coupling to a ventilator (tidal volume $450 \mathrm{~mL}$, respiratory rate $12 / \mathrm{min}$ ). Anaesthesia was maintained with infusion of propofol $(3 \mathrm{mg} / \mathrm{kg} / \mathrm{h})$ and fentanyl $(15-25 \mu \mathrm{g} / \mathrm{kg} / \mathrm{h})$. By a surgical cut down in the groin, the right common femoral artery was exposed and a $10 \mathrm{~F}$ introducer sheath was inserted into the artery. Then a bolus injection of heparin $(100 \mathrm{IU} / \mathrm{kg})$ was given through the sheath. For the balloon overstretch injury, a $12 \mathrm{~mm}$ over-the-wire angioplasty balloon was randomized to be placed in either the left or right carotid artery. The injury was induced by inflation to 5-8 atmospheres for a few seconds, which was empirically chosen to create vessel wall edema while minimizing the risk of dissection and haemorrhage. The inflated balloon was pulled gently back and forth a few millimetres before deflation to denudate the endothelium. Table 1 summarizes the experimental protocol.

\section{Magnetic resonance imaging}

The pigs were kept in general anaesthesia and one to seven hours (average four hours) after the balloon injury, the carotid CMR protocol was performed on a $1.5 \mathrm{~T}$ system (Achieva, Philips Healthcare, Best, The Netherlands) using a five channel sense cardiac coil. All the pigs were imaged in the supine position. A standardized imaging protocol was applied. After an initial localizer scan, an axial 2D time-of-flight CMR angiography was conducted

Table 1 Overview of experimental protocol

\begin{tabular}{cccccc}
\hline Pig no. & $\begin{array}{c}\text { Carotid artery } \\
\text { randomized for } \\
\text { balloon injury }\end{array}$ & $\begin{array}{c}\text { Balloon size } \\
\text { (diameter in } \mathbf{~ m m} \text { ) }\end{array}$ & $\begin{array}{c}\text { Applied } \\
\text { pressure (atm) }\end{array}$ & $\begin{array}{c}\text { Hours after balloon injury } \\
\text { when MR scanning } \\
\text { was performed }\end{array}$ & $\begin{array}{c}\text { Minutes of Evans Blue } \\
\text { circulation after 45 minutes } \\
\text { of infusion }\end{array}$ \\
\hline 1 & Right & 12 & 8 & 5 & 60 \\
2 & Right & 10 & 12 & 6 & 90 \\
3 & Left & 12 & 7 & 7 & 90 \\
5 & Left & 12 & 6 & 2 & 90 \\
6 & Left & 12 & 6 & 6 & 90 \\
7 & Right & 12 & 6 & 1 & 90 \\
8 & Right & 12 & 7 & 1 & 90 \\
9 & Right & 12 & 6 & 3,5 & 90 \\
\hline
\end{tabular}

Pig no. 4 developed dissection and occlusion of the right carotid artery, so the MR images of this pig were withdrawn from further data analysis. Thus, eight pigs were included in the analysis. To compensate for the slightly smaller balloon (10 mm) used for pig no. 2 , a higher pressure was applied to get the same amount of dilation as estimated on x-ray angiography. 
to identify the carotid arteries. Then the three blackblood imaging sequences were all acquired in the axial plane according to scanning parameters summarized in Table 2. The field of view (FOV) covered the carotid arteries from the common carotid trunk to a couple of centimeters above the carotid bifurcation. The edema scanning was performed using 2D T2-weighted shorttau inversion recovery (T2-STIR) [24], 3D volumetric isotropic turbo spin echo acquisition (VISTA) [23] and 3D T2 prepared gradient-echo (T2prep-GE) [25] with motion sensitized gradients to ensure black-blood properties [26] and multi-echo 2 point DIXON for fat-water separation less sensitive to B0 inhomogeneities compared to spectral selective saturation prepulses [27]. The Motion-Sensitized Driven-Equilibrium (MSDE) Velocity Encoding (VENC) value was set to $2.1 \mathrm{~cm} / \mathrm{s}$ in the sliceselect direction. For the VISTA sequence, the variableflip-angle refocusing pulse train was used, with $\alpha_{\min }$ of $18^{\circ}$ and $\alpha_{\max }$ of $120^{\circ}$. Cardiac triggering was used for the T2-STIR and T2prep-GE sequences to obtain images in the mid-diastolic phase. Constant level appearance (CLEAR) was used for the data acquisition of all three imaging sequences. Since CLEAR alters the noise appearance by creating a constant intensity level on the entire image, SNR should be considered an estimate. However, over- or underestimation of the SNR is likely to be within the same range for all three sequences and therefore it should not affect the statistical comparisons.

\section{Evans blue dye}

Immediately after completion of the CMR examination, the pigs were taken back to the surgical facility. Evans

Table 2 Imaging parameters for T2-STIR, VISTA, and T2prep-GE sequences

\begin{tabular}{llll}
\hline $\begin{array}{l}\text { MRI sequence } \\
\text { Technique }\end{array}$ & $\begin{array}{l}\text { T2-STIR } \\
\text { 2D black } \\
\text { blood, TSE }\end{array}$ & $\begin{array}{l}\text { VISTA } \\
\text { 3D black } \\
\text { blood, TSE }\end{array}$ & $\begin{array}{l}\text { 32prep-GE } \\
\text { blood, GE }\end{array}$ \\
\hline TE, ms & 100 & 366 & $1.88 / 3.5$ \\
TR, ms & 1800 & 1400 & 5.9 ms \\
Echo train length & 30 & 177 & - \\
Matrix size & $200 \times 200$ & $200 \times 200$ & $200 \times 200$ \\
Number of slices & 50 & 397 & 396 \\
Spatial resolution, mm & $1.5 \times 1.57 \times 4$ & $1 \times 1 \times 1$ & $1 \times 1 \times 1$ \\
Slice gap, mm & 4 & - & - \\
Number of signal & 2 & 4 & 2 \\
average (NSA) & & - & 15 \\
Flip angle & 90 & - & 16 \\
TFE factor & - & 16 & 17 \\
Scan time, minutes & 24 & & \\
\hline
\end{tabular}

T2-STIR; T2-weighted Short Tau Inversion Recovery. VISTA; Volumetric ISotropic Turbo spin echo Acquisition. T2prep-GE; T2 prepared gradient echo. TSE; Turbo Spin Echo. TFE; Turbo Field Echo.
Blue dye (EBD) was used to validate the CMR findings of vessel wall edema by identifying the location of the balloon-injured segment of the artery ex vivo and verifying macroscopically that the balloon injury had indeed increased the permeability of the artery. After intravenous injection, the dye binds to albumin. Only in case of a defect endothelial barrier, albumin with attached EBD will enter the vessel wall and the surrounding tissue. Injury to the vessel wall, however, increases the vessel wall permeability and allows albumin to leak out of the vessel together with edema-forming fluid. The EBD solution was made by dissolving $2 \mathrm{~g}$ of EBD in $50 \mathrm{~mL}$ of saline. EBD was administered intravenously during $45 \mathrm{~min}$. and then circulated in the bloodstream for additional $90 \mathrm{~min}$. In pig no.1, EBD only circulated for $60 \mathrm{~min}$., which was increased to $90 \mathrm{~min}$. for the remaining pigs to increase the wash out of EBD. Afterwards the pigs were euthanized by an overdose of pentobarbital and the carotid arteries were harvested. The carotid arteries were fixated in formaldehyde and kept in phosphate buffer for a few days until further examination. To register any possible uptake of EBD, the carotid arteries were cut open longitudinally and photographed with a digital camera (Nikon, Tokyo, Japan).

\section{Data analysis \\ Cardiovascular magnetic resonance}

The luminal and outer arterial wall boundary were delineated manually by drawing regions of interest (ROIs) on the T2-STIR, VISTA and T2prep-GE images using Osirix version 5.0.2. The vessel wall was determined by subtracting the inner luminal ROI from the outer ROI. The segmented area covered the injured and noninjured carotid arteries from the common carotid trunk to the carotid bifurcation. The CMR images were graded as 0 (non-diagnostic), 1 (poor), 2 (fair), 3 (good) or 4 (excellent) according to the delineation of the vessel wall and overall image quality. Images graded as 0 or 1 were discarded from further analysis and typically included images in the outer parts of the FOV at the borders of the coil range. For the T2-STIR sequence, 98.6\% (281/285) of the segmented slices were graded $\geq 2$, for the VISTA sequence it was $97.2 \%(1976 / 2032)$ and for T2prep-GE it was $92.2 \%(1823 / 1977)$ of the segmented slices. A reference ROI was placed in a homogenous region of corresponding muscles on the two sides, mostly the sternocleidomastoid muscle. However, this muscle was not present in images in the outer parts of the FOV, and in this case corresponding muscles of the lateral or posterior neck was used. A reference ROI was also placed in the image background on both sides. The normalized signal intensity $\left(\mathrm{SI}_{\text {mean }}\right)$ of the vessel wall on each side was calculated as $\mathrm{SI}_{\text {vessel wall }} / \mathrm{SI}_{\text {muscle }}$ for each slice and an average value was calculated for each carotid vessel. Contrast to noise ratio (CNR) was 
calculated for the T2-STIR, VISTA and T2prep-GE images according to the signal to noise ratios (SNR) of injured and non-injured carotid artery:

$$
\begin{aligned}
\mathrm{CNR} & =\mathrm{SNR}_{\text {injured }}-\mathrm{SNR}_{\text {non-injured }}, \\
\mathrm{SNR} & =\mathrm{SI}_{\text {mean }} / \mathrm{SD}_{\text {noise }}
\end{aligned}
$$

Thus, the SNR was calculated as the ratio between signal intensity of the vessel wall and signal intensity of muscle times the $\mathrm{SD}_{\text {noise }}$ to ensure independence of different anatomical positions relative to the surface coil. All slices were segmented by one observer (LØB) who was blinded to the injury status. To test the influence of variation in vessel wall segmentation, all outer ROI's were increased by $10 \%$ in all slices since this was often the most difficult ROI to place correctly due to adjacent anatomical structures such as the thyroid gland and lymph nodes with iso-intense SI or edema leaking out of the vessel into the surroundings, cf. Table 3. An in-house MATLAB R2012b script was developed to extract ROI data and to perform statistical analysis. An additional script used ROI data to produce in-plane images of the carotid arteries.

\section{Evans blue dye}

The digital photos of the carotid arteries were reviewed by two observers (LØB, ESSH) for uptake of EBD. The relative distances between anatomical landmarks were found by segmentation of the photos in Osirix version 5.0.2.

The CMR images of the injured and non-injured carotid arteries were matched to the macroscopic staining by EBD of the carotid arteries for each of the three CMR sequences using anatomical landmarks, such as the distance from the common carotid trunk and the upper carotid bifurcation as well as gross morphological features of the lumen and vessel wall.

\section{Statistical analysis}

Each pig was considered a paired set of data consisting of an injured and non-injured carotid artery. Student's paired $t$-test was used to compare SNR between injured and non-injured vessel. SNR and CNR for all three imaging sequences are presented as mean $+95 \%$ confidence intervals $\left(\mathrm{CI}_{95}\right)$. We compared differences in SNR and $\mathrm{CNR}$ between the sequences using one-way analysis of

Table 3 Results for enlarged ROls to test for the importance of the accuracy of segmentation

\begin{tabular}{llllll}
\hline $\begin{array}{l}\text { Imaging } \\
\text { sequence }\end{array}$ & $\begin{array}{l}\text { Paired t-test } \\
\mathbf{p} \text {-value }\end{array}$ & $\mathbf{C l}_{\mathbf{9 5}}$ & SNR $_{\text {injured }}$ & $\mathbf{S N R}_{\text {non-injured }}$ & CNR \\
\hline T2STIR & 0.005 & $8.05-29.9$ & 23.0 & 17.9 & 5.1 \\
VISTA & 0.009 & $5.5-27.7$ & 24.3 & 19.0 & 5.4 \\
T2prep-GE & 0.006 & $4.4-18.0$ & 21.5 & 17.3 & 4.2 \\
\hline
\end{tabular}

variance (ANOVA). All calculations were made using MATLAB R2012b Statistics Toolbox.

\section{Results}

All CMR scans were performed successfully. All three imaging sequences classified the injured and non-injured carotid artery correctly according to the EBD staining of the carotid arteries and demonstrated a significant increase in the SNR of the vessel wall in the injured compared to the non-injured carotid artery (Figure 1). The results of the paired t-tests and SNR and CNR for all three imaging sequences are presented in Table 4. Typical images of the carotid arteries showing STIR, VISTA and T2prep-GE images together with the corresponding EBD image are shown in Figure 2.

ANOVA showed no significant differences in SNR at the $\mathrm{p}<0.05$ level for the three sequences neither for the injured carotid artery $[F(2,21)=0.38, p=0.6917]$ nor for the non-injured carotid artery $[\mathrm{F}(2,21)=0.19, \mathrm{p}=0.8315]$. ANOVA for CNR between the three sequences showed no significant differences $[F(2,21)=0.3, p=0.7447]$. Enlargement by $10 \%$ of all ROIs delineating the outer carotid wall did not change the significance of the results as is seen from Table 3.

\section{Discussion}

In this study, we have demonstrated the ability of the 3D CMR sequences VISTA and T2prep-GE to visualize vessel wall edema in porcine carotid arteries following acute

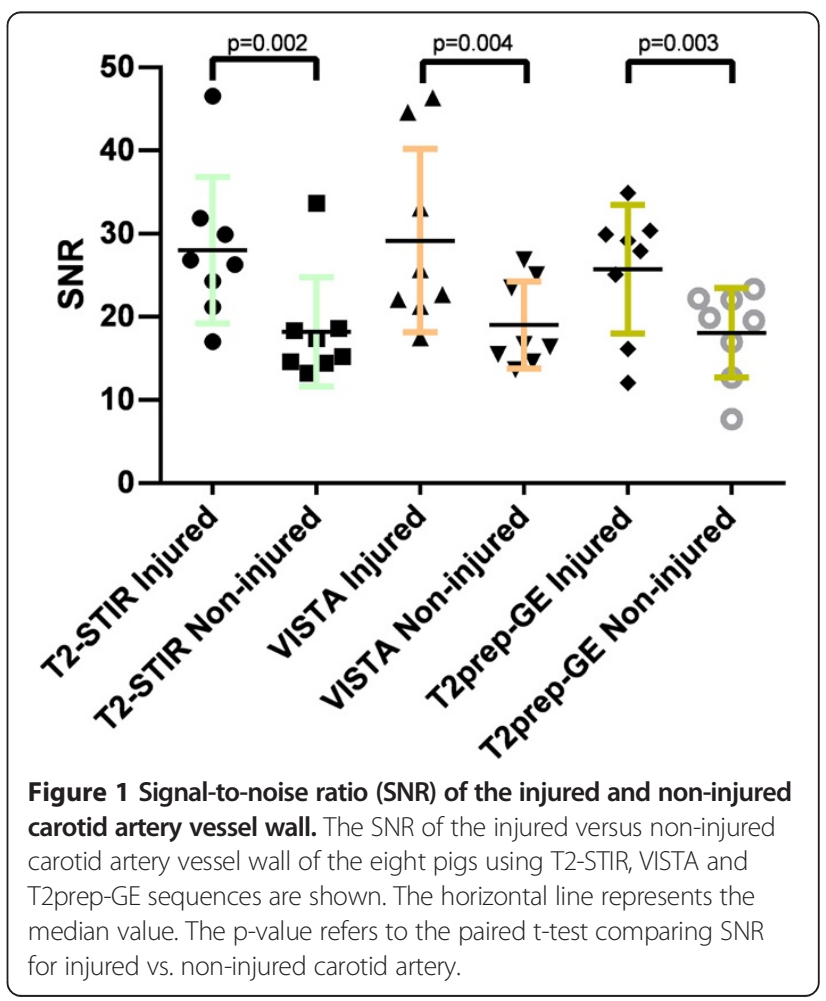


Table 4 SNR and CNR values for T2-STIR, VISTA and T2prep-GE

\begin{tabular}{ccccccc}
\hline & Paired t-test $\mathbf{p}$-value & Mean difference & $\mathbf{C l}_{\mathbf{9 5}}$ & SNR $_{\text {injured }}$ & SNR $_{\text {non-injured }}$ & CNR \\
\hline T2-STIR & 0.002 & 9.8 & {$[6.9-12,8]$} & $22.5,[16.8-28.2]$ & $17.3,[12.4-22.3]$ & $5.15,[3.0-7.3]$ \\
VISTA & 0.004 & 6.9 & {$[4.4-15.9]$} & $23.8,[17.1-30.6]$ & $18.3,[14.2-22.3]$ & $5.6,[1.7-9.5]$ \\
T2prep-GE & 0.003 & 4.6 & {$[3.8-11.5]$} & $20.9,[17.0-24.9]$ & $16.7,[12.8-20.5]$ & $4.3,[1.9-6.7]$ \\
\hline
\end{tabular}

The $p$-value refers to the paired t-test comparing SNR for injured vs. non-injured carotid artery.

balloon injury. A significant increase in SI within the injured compared to the non-injured carotid artery vessel wall was observed, which was comparable to the conventional 2D T2-STIR imaging sequence. Due to the inherent higher SNR, the 3D sequences allowed for $1 \mathrm{~mm}^{3}$ isotropic spatial resolution with a shorter scan time compared to $2 \mathrm{D}$ T2-STIR.

To the best of our knowledge, this is the first systematic comparison of $2 \mathrm{D}$ and $3 \mathrm{D}$ imaging sequences for the visualization of vessel wall edema with implications for further clinical studies to unravel the importance of plaque edema in the natural history of vulnerable atherosclerotic phenotype.

Pedersen et al. have shown that gadofosveset, an albumin-binding gadolinium contrast agent, detects endothelial damage and angiogenesis in this experimental model of vessel wall edema [28]. Phinikaridou et al. demonstrated the presence of gadofosveset in the vessel wall of a murine atherosclerosis model [29]. Uptake of gadofosveset correlated with EBD staining, morphological changes of endothelial cells, and widening of cell-cell junctions measured by electron microscopy suggesting an increased

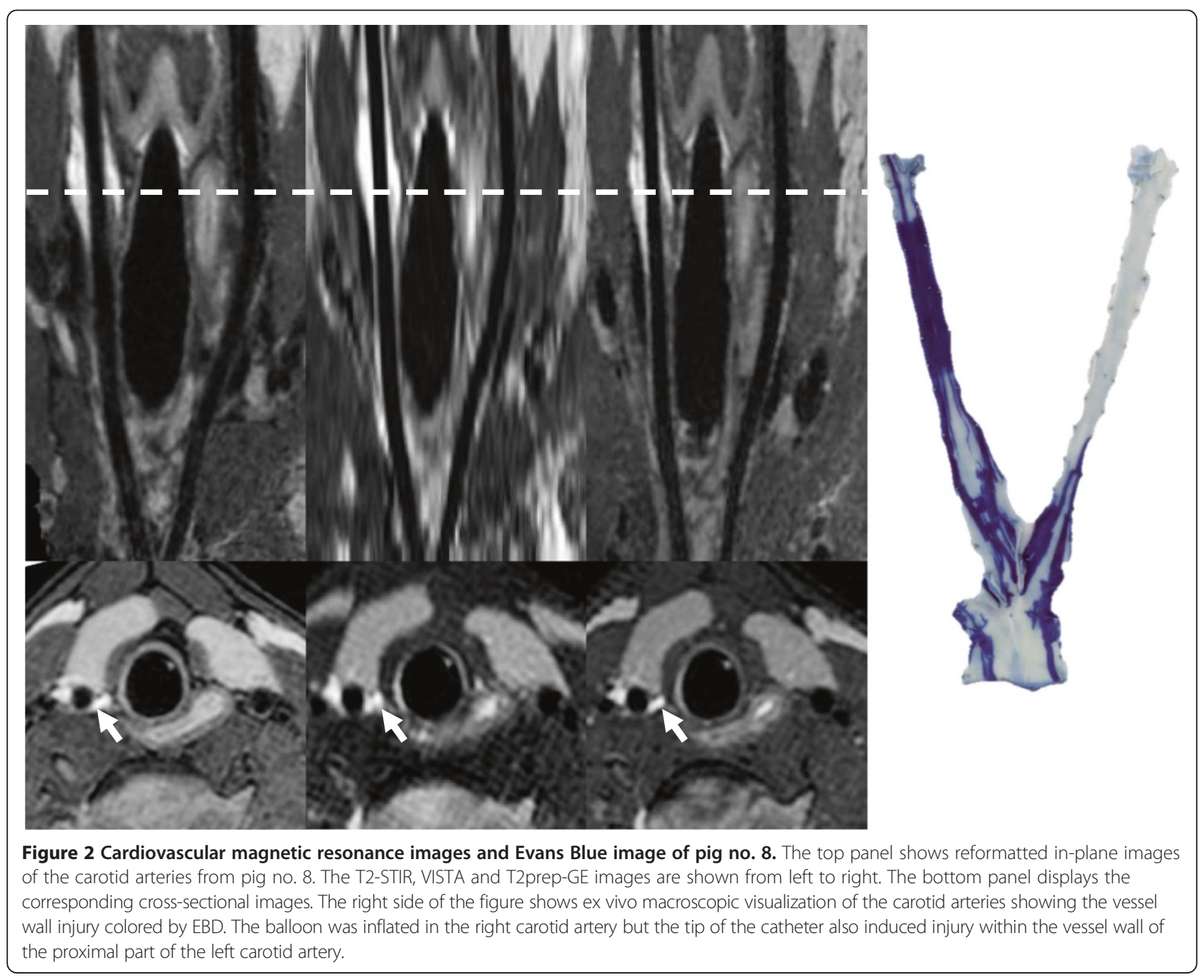


permeability [29]. Also, Pedersen et al. used fibrinogen as a surrogate marker of edema with the same rationale as described for albumin and demonstrated a linear correlation between the area of fibrinogen staining on immunohistochemistry and the relative SI on CMR $(\rho=0.93, \mathrm{P}<0.001)$ and also demonstrated agreement between hyperintense SI on T2-STIR images and uptake of EBD $\left(\mathrm{X}^{2}=17.14, \mathrm{P}<0.001\right)$ [19]. Thus, balloon overstretch injury is a validated experimental method causing vessel wall edema. Since it is difficult to histologically verify edema, EBD is used experimentally to assess vessel wall permeability and edema $[18,19,29]$. In this study, we detected the macroscopic presence of EBD in the vessel wall to locate the balloon injury and used this as reference in the comparison of the CMR sequences. The mechanical balloon injury to the vessel wall was twofold as both overstretching of the vessel wall and pulling the deflated balloon backwards will inevitably cause injury to the vessel wall. Consequently, all carotid segments exposed to the balloon or just the catheter itself demonstrated uptake of EBD. This is demonstrated in the EBD image for pig no. 8 in Figure 2 where the tip of catheter had also been placed in the proximal part of the non-injured artery. Interestingly, it seems that endothelial denudation is enough to locally increase vessel wall permeability, as has been suggested in previous studies [28,29].

It has been shown that plaque rupture can occur also in low-grade stenosis and that the degree of luminal stenosis is not an optimal predictor of future events [30]. In carotid atherosclerosis, significant luminal stenosis may predict one out of four strokes in symptomatic patients [31] but only one out of ten strokes in asymptomatic patients [32]. Angiography methods may underestimate the atherosclerotic burden due to expansive arterial remodelling [33] and by measuring the luminal stenosis relative to the supposedly normal adjacent sites [30]. Nevertheless, luminal carotid artery stenosis remains the primary criteria for surgical therapy in symptomatic patients. CMR is emerging as a comprehensive non-invasive imaging modality for the assessment of the carotid and coronary vessel wall [34-37]. Multicontrast carotid CMR is a histologically validated method for morphological plaque classification $[35,36]$. However, due to the complexity of the scanning protocols and the carotid plaque analysis, more widespread use has been limited. Our approach using heavily T2-weighted sequences to visualize plaque edema may provide a more simplified measure of plaque vulnerability. Further studies in patients with carotid atherosclerosis are needed to verify the possible clinical impact of visualizing carotid plaque edema. Improvements have been made with the use of gadolinium contrast agents detecting several features of the vulnerable plaque [38-40] as well as the use of targeted contrast agents in molecular imaging $[8,9]$. However, a non-contrast dependent method would eliminate potential contrast-related complications. CMR may also be used in the estimation of the biomechanical stresses within the plaque $[41,42]$ which may cause mechanical instability and thereby possibly influence plaque vulnerability. Thus, different mechanisms may act in concert and different imaging approaches are needed for a comprehensive evaluation of plaque vulnerability.

Our group has shown that carotid and coronary vessel wall edema can be detected using 2D T2-STIR $[18,19]$. However, clinical application using 2D TSE sequences may be hampered by the relatively low spatial resolution in the slice-select direction making 2D images more prone to partial volume effects and the need for a relatively long acquisition time to achieve sufficient SNR and/or in-plane spatial resolution [23]. In this regard, 3D imaging protocols such as the proposed VISTA and T2prep-GE sequences have some inherent advantages with intrinsic high SNR that can be utilized to yield isotropic spatial resolution and improved anatomic coverage of plaque components. Furthermore, imaging 3D isotropic volumes, multi-planar reformation allows for reformatting images in arbitrary planes, which is often helpful for depicting anatomical structures [23]. Zhang et al. reported that VISTA and 2D TSE performed similarly in measurements of wall and lumen volume and that VISTA demonstrated significantly higher CNR and markedly reduced scan time [23]. The T2prep-GE technique has been applied for cardiac magnetic resonance $[43,44]$ and the fat-water separation obtained with this sequence may also be useful in atherosclerosis imaging.

\section{Limitations}

In terms of applying these results to atherosclerosis imaging, it may be considered a limitation to study vessel wall edema in a non-atherosclerotic animal model of balloon injury. However, for validating CMR sequences, it may be considered advantageous to be able to visualize vessel wall edema in isolation. The balloon injury may cause more edema than there will be present in an atherosclerotic plaque but even very subtle injury to the vessel caused by pulling back the deflated balloon was reliably detected by CMR in accordance with uptake of EBD. Since both the thyroid gland and lymph nodes around the proximal part of the carotid arteries would appear bright on the T2W images, accurate segmentation of the carotid vessel wall was sometimes difficult. However, as seen from Table 3 even deliberately enlarging the outer manual delineation of the vessel wall did not change the significance of the results illustrating the robustness of the segmentation and comparison method of injured versus non-injured vessel.

\section{Conclusion}

We have demonstrated the ability of $3 \mathrm{D}$ T2W blackblood imaging sequences to detect vessel wall edema in 
porcine carotid arteries following acute balloon injury. Clinical application of vessel wall edema visualization in carotid atherosclerosis may benefit from 3D imaging sequences compared to conventional 2D imaging techniques due to inherently higher SNR allowing for isotropic spatial resolution. Plaque edema in atherosclerosis may prove to be a useful surrogate marker in the identification of the vulnerable plaque, and further studies are needed to determine the clinical applicability of vessel wall edema.

\section{Abbreviations}

2D: Two-dimensional; 3D: Three-dimensional; a: Flip angle; CLEAR: Constant level appearance; CMR: Cardiovascular magnetic resonance; CNR: Contrast-tonoise-ratio; EBD: Evans blue dye; FOV: Field of view; MSDE: Motion-sensitized driven-equilibrium; T2pre-GE: T2 prepared gradient-echo; RF: Radio frequency; ROI: Region of interest; SI: Signal intensity; SNR: Signal-to-noise-ratio; STIR: Short tau inversion recovery; T2W: T2-weighted; TE: Echo time; TI: Inversion time; TR: Repetition time; TSE: Turbo spin echo; VENC: Velocity encoding value; VISTA: Volumetric isotropic turbo spin echo acquisition.

\section{Competing interests}

The authors declare that they have no competing interests.

\section{Authors' contributions}

$\llcorner\varnothing B$ carried out all the procedures related to the animal model, contributed to performing all the CMR scans, analyzed the acquired CMR data and histological data, contributed to the statistical analyses as well as elaboration of all figures and tables, wrote the manuscript, and merged all feedback from the co-authors into the final manuscript. AYKGH contributed to procedures related to the animal model, and drafting the manuscript and revised it critically for intellectual content. SFP contributed to the procedures related to the animal model and formulating the study design, and drafting the manuscript and revised it critically for intellectual content. JLH contributed to the study design, and drafting the manuscript and revised it critically for intellectual content. WYK took part in formulating the study design, the CMR sequence setup, drafting the manuscript and revised it critically and contributed to important intellectual content of the manuscript. ESSH contributed to the CMR sequence development and to performing the CMR scans as well as the analyses of the acquired CMR data and histological data, the statistical analyses, the figures and tables, drafting the manuscript and revised it critically for intellectual content. All authors have read and approved the final manuscript.

\section{Acknowledgements}

The work was made possible with grants from Novo Nordisk foundation, Master Cabinetmaker Sophus Jacobsen \& Wife Astrid Jacobsen's Foundation. The work was performed at Institute of Clinical Medicine and MR Research Centre, Aarhus University Hospital Skejby, Brendstrupgaardsvej 100, DK-8200 Aarhus N, Denmark.

\section{Author details}

'Department of Cardiology, Aarhus University Hospital Skejby, Brendstrupgaardsvej 100, DK-8200 Aarhus N, Denmark. ${ }^{2}$ MR Research Centre, Aarhus University Hospital Skejby, Brendstrupgaardsvej 100, DK-8200 Aarhus N, Denmark. ${ }^{3}$ Department of Cardiothoracic \& Vascular Surgery, Aarhus University Hospital Skejby, Brendstrupgaardsvej 100, DK-8200 Aarhus N, Denmark.

Received: 28 September 2013 Accepted: 10 February 2014 Published: 4 March 2014

\section{References}

1. Go AS, Mozaffarian D, Roger VL, Benjamin EJ, Berry JD, Blaha MJ, Dai S, Ford ES, Fox CS, Franco S, et al. Heart disease and stroke statistics-2014 update: a report from the american heart association. Circulation. 2014; 129:e28-292.

2. Fisher $\mathrm{M}$, Folland $\mathrm{E}$. Acute ischemic coronary artery disease and ischemic stroke: similarities and differences. Am J Ther. 2008; 15:137-49.

3. Shah PK. Inflammation and plaque vulnerability. Cardiovasc Drugs Ther. 2009; 23:31-40.
4. Thim T, Hagensen MK, Bentzon JF, Falk E. From vulnerable plaque to atherothrombosis. J Intern Med. 2008; 263:506-16.

5. Rudd JH, Warburton EA, Fryer TD, Jones HA, Clark JC, Antoun N, Johnstrom P, Davenport AP, Kirkpatrick PJ, Arch BN, Pickard JD, Weissberg PL. Imaging atherosclerotic plaque inflammation with [18F]-fluorodeoxyglucose positron emission tomography. Circulation. 2002; 105:2708-11.

6. Tawakol A, Migrino RQ, Hoffmann U, Abbara S, Houser S, Gewirtz H, Muller JE, Brady TJ, Fischman AJ. Noninvasive in vivo measurement of vascular inflammation with F-18 fluorodeoxyglucose positron emission tomography. J Nucl Cardiol. 2005; 12:294-301.

7. Tawakol A, Migrino RQ, Bashian GG, Bedri S, Vermylen D, Cury RC, Yates D, LaMuraglia GM, Furie K, Houser S, Gewirtz H, Muller JE, Brady TJ, Fischman AJ. In vivo 18F-fluorodeoxyglucose positron emission tomography imaging provides a noninvasive measure of carotid plaque inflammation in patients. J Am Coll Cardiol. 2006; 48:1818-24.

8. Ronald JA, Chen JW, Chen Y, Hamilton AM, Rodriguez E, Reynolds F, Hegele RA, Rogers KA, Querol M, Bogdanov A, Weissleder R, Rutt BK. Enzymesensitive magnetic resonance imaging targeting myeloperoxidase identifies active inflammation in experimental rabbit atherosclerotic plaques. Circulation. 2009; 120:592-9.

9. Makowski MR, Wiethoff AJ, Blume U, Cuello F, Warley A, Jansen CH, Nagel E, Razavi R, Onthank DC, Cesati RR, Marber MS, Schaeffter T, Smith A, Robinson SP, Botnar RM. Assessment of atherosclerotic plaque burden with an elastinspecific magnetic resonance contrast agent. Nat Med. 2011; 17:383-8.

10. Trivedi RA, Mallawarachi C, King-Im JM, Graves MJ, Horsley J, Goddard MJ, Brown A, Wang L, Kirkpatrick PJ, Brown J, Gillard JH. Identifying inflamed carotid plaques using in vivo USPIO-enhanced MR imaging to label plaque macrophages. Arterioscler Thromb Vasc Biol. 2006; 26:1601-6.

11. Howarth SP, Tang TY, Trivedi R, Weerakkody R, King-Im J, Gaunt ME, Boyle JR, Li ZY, Miller SR, Graves MJ, et al. Utility of USPIO-enhanced MR imaging to identify inflammation and the fibrous cap: a comparison of symptomatic and asymptomatic individuals. Eur J Radiol. 2009; 70:555-60.

12. Tearney GJ, Yabushita H, Houser SL, Aretz HT, Jang IK, Schlendorf KH Kauffman CR, Shishkov M, Halpern EF, Bouma BE. Quantification of macrophage content in atherosclerotic plaques by optical coherence tomography. Circulation. 2003; 107:113-9.

13. Moreno PR, Lodder RA, Purushothaman KR, Charash WE, O'Connor WN, Muller JE. Detection of lipid pool, thin fibrous cap, and inflammatory cells in human aortic atherosclerotic plaques by near-infrared spectroscopy. Circulation. 2002; 105:923-7.

14. Casscells W, Hathorn B, David M, Krabach T, Vaughn WK, McAllister HA, Bearman G, Willerson JT. Thermal detection of cellular infiltrates in living atherosclerotic plaques: possible implications for plaque rupture and thrombosis. Lancet. 1996; 347:1447-51.

15. Stefanadis C, Diamantopoulos L, Vlachopoulos C, Tsiamis E, Dernellis J, Toutouzas K, Stefanadi E, Toutouzas P. Thermal heterogeneity within human atherosclerotic coronary arteries detected in vivo: a new method of detection by application of a special thermography catheter. Circulation. 1999: 99:1965-71.

16. Falk E. Pathogenesis of atherosclerosis. J Am Coll Cardiol. 2006; 47:C7-12.

17. Libby P. Inflammation in atherosclerosis. Nature. 2002; 420:868-74.

18. Pedersen SF, Thrysoe SA, Paaske WP, Thim T, Falk E, Ringgaard S, Kim WY. Determination of edema in porcine coronary arteries by $\mathrm{T} 2$ weighted cardiovascular magnetic resonance. I Cardiovasc Magn Reson. 2011; 13:52.

19. Pedersen SF, Kim WY, Paaske WP, Thim T, Falk E, Ringgaard S, Thrysoe SA. Determination of acute vascular injury and edema in porcine carotid arteries by $\mathrm{T} 2$ weighted cardiovascular magnetic resonance. Int $\rfloor$ Cardiovasc Imaging. 2012; 28:1717-24

20. Kim WY, Christiansen EH, Thrysoe SA, Al-Mashhadi RH, Botker HE, Bottcher $M$, Holm NR, Falk E. First in vivo demonstration of coronary edema in culprit lesion of patient with acute coronary syndrome by cardiovascular magnetic resonance. Circ Cardiovasc Imaging. 2011; 4:344-6.

21. Holm NR, Kim WY, Maeng M, Thrysoe SA, Botker HE, Thuesen L, Hoyer S, Tu S, Falk E, Lassen JF, et al. Coronary edema demonstrated by cardiovascular magnetic resonance in patients with peri-stent inflammation and aneurysm formation after treatment by drug-eluting stents. Circ Cardiovasc Imaging. 2013; 6:352-4.

22. Pels K, Labinaz M, Hoffert C, O'Brien ER. Adventitial angiogenesis early after coronary angioplasty: correlation with arterial remodeling. Arterioscler Thromb Vasc Biol. 1999; 19:229-38. 
23. Zhang Z, Fan Z, Carroll TJ, Chung Y, Weale P, Jerecic R, Li D. Three-dimensional T2-weighted MRI of the human femoral arterial vessel wall at 3.0 Tesla. Invest Radiol. 2009; 44:619-26.

24. Simonetti OP, Finn JP, White RD, Laub G, Henry DA. "Black blood" T2-weighted inversion-recovery MR imaging of the heart. Radiology. 1996; 199:49-57.

25. Botnar RM, Stuber M, Danias PG, Kissinger KV, Manning WJ. Improved coronary artery definition with T2-weighted, free-breathing three-dimensional coronary MRA. Circulation. 1999; 99:3139-48.

26. Wang J, Yarnykh VL, Hatsukami T, Chu B, Balu N, Yuan C. Improved suppression of plaque-mimicking artifacts in black-blood carotid atherosclerosis imaging using a multislice motion-sensitized driven-equilibrium (MSDE) turbo spin-echo (TSE) sequence. Magn Reson Med. 2007; 58:973-81.

27. Henningsson M, Boernert $P$, Koken $P$, Botnar RM. Fat suppression for coronary MR angiography at 3T: 2 point Dixon versus Spectral Presaturation with Inversion Recovery (SPIR). J Cardiovasc Magn Reson. 2013; 15:E9.

28. Pedersen SF, Thrysoe SA, Paaske WP, Thim T, Falk E, Ringgaard S, Kim WY. CMR assessment of endothelial damage and angiogenesis in porcine coronary arteries using gadofosveset. J Cardiovasc Magn Reson. 2011; 13:10.

29. Phinikaridou A, Andia ME, Protti A, Indermuehle A, Shah A, Smith A Warley A, Botnar RM. Noninvasive magnetic resonance imaging evaluation of endothelial permeability in murine atherosclerosis using an albumin-binding contrast agent. Circulation. 2012; 126:707-19.

30. Wasserman BA, Wityk RJ, Trout HH III, Virmani R. Low-grade carotid stenosis: looking beyond the lumen with MRI. Stroke. 2005; 36:2504-13.

31. North American Symptomatic Carotid Endarterectomy Trial Collaborators. Beneficial effect of carotid endarterectomy in symptomatic patients with high-grade carotid stenosis. N Engl J Med. 1991; 325:445-53.

32. Executive Committee for the Asymptomatic Carotid Atherosclerosis Study. Endarterectomy for asymptomatic carotid artery stenosis. JAMA. 1995; 273:1421-8.

33. Keenan NG, Pennell DJ, Mohiaddin RH. Glagov remodelling in the atherosclerotic carotid artery by cardiovascular magnetic resonance. Heart. 2008; 94:228.

34. Botnar RM, Stuber M, Kissinger KV, Kim WY, Spuentrup E, Manning WJ. Noninvasive coronary vessel wall and plaque imaging with magnetic resonance imaging. Circulation. 2000; 102:2582-7.

35. Cai JM, Hatsukami TS, Ferguson MS, Small R, Polissar NL, Yuan C. Classification of human carotid atherosclerotic lesions with in vivo multicontrast magnetic resonance imaging. Circulation. 2002; 106:1368-73.

36. Yuan C, Mitsumori LM, Ferguson MS, Polissar NL, Echelard D, Ortiz G, Small R, Davies JW, Kerwin WS, Hatsukami TS. In vivo accuracy of multispectral magnetic resonance imaging for identifying lipid-rich necrotic cores and intraplaque hemorrhage in advanced human carotid plaques. Circulation. 2001: 104:2051-6.

37. Takaya N, Yuan C, Chu B, Saam T, Polissar NL, Jarvik GP, Isaac C, McDonough J, Natiello C, Small R, Ferguson MS, Hatsukami TS. Presence of intraplaque hemorrhage stimulates progression of carotid atherosclerotic plaques: a high-resolution magnetic resonance imaging study. Circulation. 2005; 111:2768-75.

38. Cai J, Hatsukami TS, Ferguson MS, Kerwin WS, Saam T, Chu B, Takaya N, Polissar NL, Yuan C. In vivo quantitative measurement of intact fibrous cap and lipid-rich necrotic core size in atherosclerotic carotid plaque: comparison of high-resolution, contrast-enhanced magnetic resonance imaging and histology. Circulation. 2005; 112:3437-44.

39. Millon A, Boussel L, Brevet M, Mathevet JL, Canet-Soulas E, Mory C, Scoazec JY, Douek P. Clinical and histological significance of gadolinium enhancement in carotid atherosclerotic plaque. Stroke. 2012; 43:3023-8.

40. Yuan C, Kerwin WS, Ferguson MS, Polissar N, Zhang S, Cai J, Hatsukami TS. Contrast-enhanced high resolution MRI for atherosclerotic carotid artery tissue characterization. J Magn Reson Imaging. 2002; 15:62-7.

41. Tang TY, Howarth SP, Li ZY, Miller SR, Graves MJ, King-Im JM, Trivedi RA, Walsh SR, Brown AP, Kirkpatrick PJ, et al. Correlation of carotid atheromatous plaque inflammation with biomechanical stress: utility of USPIO enhanced MR imaging and finite element analysis. Atherosclerosis. 2008; 196:879-87.
42. Sadat U, Teng Z, Young VE, Graves MJ, Gaunt ME, Gillard JH. High-resolution magnetic resonance imaging-based biomechanical stress analysis of carotid atheroma: a comparison of single transient ischaemic attack, recurrent transient ischaemic attacks, non-disabling stroke and asymptomatic patient groups. Eur J Vasc Endovasc Surg. 2011; 41:83-90.

43. Farrelly C, Shah S, Davarpanah A, Keeling AN, Carr JC. ECG-gated multiecho Dixon fat-water separation in cardiac MRI: advantages over conventional fat-saturated imaging. AJR Am J Roentgenol. 2012; 199:W74-83.

44. Kellman P, Hernando D, Shah S, Zuehlsdorff S, Jerecic R, Mancini C, Liang ZP, Arai AE. Multiecho dixon fat and water separation method for detecting fibrofatty infiltration in the myocardium. Magn Reson Med. 2009; 61:215-21.

\section{doi:10.1186/1532-429X-16-22}

Cite this article as: Bloch et al:: Imaging of carotid artery vessel wall edema using T2-weighted cardiovascular magnetic resonance. Journal of Cardiovascular Magnetic Resonance 2014 16:22.

\section{Submit your next manuscript to BioMed Central and take full advantage of:}

- Convenient online submission

- Thorough peer review

- No space constraints or color figure charges

- Immediate publication on acceptance

- Inclusion in PubMed, CAS, Scopus and Google Scholar

- Research which is freely available for redistribution 